\title{
Tecnología, ingeniería y ciclos de formación
}

\section{Technology, engineering and formation cycles}

\author{
Mirna Jirón Popova \\ Candidata a Doctora en Educación, Magíster en Educación, Magíster en Inge- \\ niería Mecánica, Ing. Mecánico. Docente Asociado de la Universidad Distrital \\ Francisco José de Caldas. Bogotá, Colombia. \\ Contacto:mjiron@udistrital.edu.co. \\ Fecha de recepción: 30 de junio de 2013 \\ Clasificación del artículo: investigación \\ Fecha de aceptación: 27 de agosto de 2013 \\ Financiamiento: Universidad Distrital Francisco José de Caldas
}

Palabras clave: Colombia, educación superior formación de ingeniero, formación por ciclos formación tecnológica

Key words: Colombia, Engineers Formation, Formation by Cycles, Higher Education, Technological Formation

\section{RESUMEN}

Este artículo presenta parte de los resultados de la investigación La formación tecnológica en Colombia en el ámbito de la universidad pública, particularmente en la Universidad Distrital Francisco José de Caldas, que fue una de las instituciones observadas. En este artículo se exponen los acontecimientos que se suceden desde la institucionalización de la Facultad Tecnológica, con miras a ofrecer Educación Superior Tecnológica a sectores desfavorecidos de la población del Distrito Capital. Se hace especial énfasis en aspectos relacionados con las tensiones entre resistir o adaptarse a lo establecido en los dispositivos de control; coyuntura que finalmente determina que la facultad trasforme su propuesta original de Educación Tecnológica por Ciclos, hacia una de Formación de Ingenieros por Ciclos y, posteriormente, por la Formación de Ingenieros por Ciclos Propedéuticos.

\section{ABSTRACT}

This article presents part of the results of the research work titled "Technological formation in Colombia in the public university field", particularly at Universidad Distrital Francisco José de Caldas, which was one of the observed institutions. Consequently, we present the findings related to the events that take place from the moment in which Technological Faculty is institutionalized, aiming at offering Technological Higher Education to disadvantaged sectors from the population at Distrito Capital. Special emphasis is made on aspects related to the tensions among resisting or adapting to the regulations of the control mechanisms; conjunction that lastly determines that this faculty would transform its original proposal of Technological Education by Cycles, towards an Engineers Formation by Cycles and, afterwards, to Engineers Formation by Propaedeutic Cycles. 


\section{investigación}

\section{INTRODUCCIÓN}

A partir de la segunda mitad del siglo XX son significativos los cambios que se suceden en el ámbito de la educación superior (en adelante: ES) en la región y en el país. De allí, el interés de los investigadores de disponer de un acervo de conocimiento sobre los acontecimientos relacionados con la modernización de la ES y, con ello, la emergencia de nuevas modalidades de formación, entre ellas de la formación tecnológica (en adelante: FT). El discurso hegemónico de la norma (Decreto 2566 de 1976, Decreto-ley 080 de 1980, Ley 30 de 1992) redujo el concepto de FT a un nivel inferior con respecto a la formación tradicional - ingeniería - a una formación de menor estatus académico y social y, por ello, una segunda opción para quienes no pueden ingresar a una carrera tradicional y se ven obligados a optar por una formación de menor duración, con menores exigencias académicas y menos rigor científico, en suma, por una carrera corta.

El país recibe los años 2000 con importantes acontecimientosnormativos relacionados con el despliegue del sistema de aseguramiento de la calidad de la ES y la reglamentación de la formación por ciclos propedéuticos (en adelante: $\mathrm{FpCP}$ ) - como se denomina a la formación por ciclos (en adelante: FpC) en Colombia-. Con respecto a la $\mathrm{FpCP}$, hay que señalar que una primera norma (Ley 749 de 2002) restringió, sin mayores justificaciones, la oferta de programas a las instituciones técnicas y tecnológicas, y a áreas de la ingeniería, tecnología de la información y administración. Un par de años después, otra ley (Ley 1188 de 2008) corrigió esa omisión y determinó que todas las instituciones de ES - incluyendo la universidad - podrían ofrecer FpCP, hasta el nivel profesional, en todos los campos y áreas del conocimiento. En el marco de esas disposiciones, la FT asume su identidad en términos de un se- gundo ciclo (Ley 749 de 2002) y de nivel tecnológico (Decreto 1295 de 2010).

Surgen entonces varias preguntas, entre las cuales está una relacionada con la indecencia de las normas anteriores sobre el concepto de FT que asumen las universidades públicas del país que ofertan programas de FT en áreas de las ingenierías. Responder esa pregunta fue una tarea que asumió el proyecto de investigación La Formación Tecnológica en Colombia en el ámbito de la universidad pública (1990-2010).

\section{METODOLOGÍA}

La investigación asumió una perspectiva histórico-hermenéutica, para comprender la confluencia de los hechos que configuran la ocurrencia de un acontecimiento e interpretar teniendo en cuenta la particularidad de los contextos de producción histórica - económico, políticos, sociales, educativos, tecnológicos, entre otros-y los posibles efectos - intereses - que estos pretendía lograr; para develar los significados y dar paso a la comprensión y reconstrucción. La metodología privilegió la observación documental, ya que ese tipo de fuente permite "estudiar la educación superior en las temáticas específicas abordadas y establecer una nueva mirada desde los acontecimientos y rupturas" (Ortíz, s.f., p. 3).

La metodología se desplegó en cuatro etapas de investigación. En la etapa documental se realizó la búsqueda de fuentes, su sistematización y obtención - impresa o digital—y, el trabajo de campo que se llevó a cabo en un conjunto de universidades públicas del país que ofrecen programas de FT, a saber: Universidad Tecnológica de Pereira (UTP), Universidad Industrial de Santander (UIS), Universidad Pedagógica y Tecnológica de Colombia (UPTC), Universidad Francisco de Paula Santander (UFPS), Universidad del Valle 


\section{investigación}

(UV) y Universidad Distrital Francisco José de Caldas (UDFJC); cuyo propósito fue ampliar el archivo documental con los testimonios de los actores sociales —coordinadores de programa o directores de escuela, profesores que participaron de la creación o conceptualización de los programas, entre otros- mediante la técnica de entrevista semiestructurada e información institucional. En la etapa textual se pasó al análisis de contenido, a las ideas globales expresadas en los documentos, con ayuda del software AtlasTi. En la etapa conceptual, se realizó la reconstrucción histórica de los acontecimientos desde la perspectiva de la universidad pública.

\section{RESULTADOS Y DISCUSIÓN}

\section{Universidad pública y formación tecnológica: a manera de antecedentes breves}

Las evidencias recogidas en el desarrollo de la investigación muestran que, a finales de los años setenta - como resultado de la presencia de las misiones internacionales $\mathrm{y}$, específicamente la misión Atcon (2009) - se motiva un proceso de modernización de la ES con miras a establecer un sistema estatificado de instituciones y programas que privilegia la expansión de modalidades de ciclo corto - para propiciar el desarrollo del país, vía industrialización - en el marco del Plan Básico de Educación Superior (1968-1975).

Atcon (2009) también recomienda que la universidad tradicional debe seguir dedicada a la oferta de programas tradicionales; sin embargo, las primeras normas relacionadas con los ciclos cortos autorizaron a dichas instituciones a ofertar ciclos cortos; considerando especialmente a las universidades regionales, sin el concurso de la cuales era poco probable cumplir con la meta de capacitar al grueso de la población; especialmente, la población ubicada en regiones alejadas de las capitales departamentales.
La FT se institucionalizó como modalidad de la ES - con la expedición del Decreto-Ley 080 de 1980 - , para el desarrollo de actividades tecnológicas que requieren investigación (Presidencia de la República, 1982). Concepto que quedó reducido a formalidades de ley, dado el carácter ocupacional con énfasis en destrezas y habilidades que en la práctica identificaba la FT; en respuesta a una industria especializada en la trasferencia de tecnologías y, por ende, en la manipulación de artefactos tecnológicos y la instrumentalización de procesos de producción, que no requerían la reflexión científica sobre las técnicas.

Son las políticas del gobierno de Belisario Betancourt - en el marco del Plan de Desarrollo Nacional Cambio con Equidad (1983-1986) - , las que impulsan los programas de FT en la universidad pública. Entonces, para algunas universidades como la UTP-, el decreto significó la renuncia - cabe decir, sin mayores resistencias-, a un proyecto académico emblemático para Risaralda, la formación de ingenieros auxiliares, de ingenieros de fábrica o de planta; que estableció la institución inspirada en los modelos de formación europeos con el propósito de ofrecer a la incipiente industria regional — de los años sesenta y setenta - personal técnico industrial; para cumplir con la política gubernamental, la institución redefine la identidad de los programas en términos de formar tecnólogos y trasforma el Instituto Politécnico y las Escuelas Auxiliares de Ingeniería en la actual Facultad de Tecnologías.

Para otras universidades (UPTC), la emergencia del decreto se tradujo en la creación de unidades especializadas para la oferta de programas de FT; bajo la estrategia de educación a distancia. Se trató del Instituto de Educación Abierta y a Distancia, hoy Facultad de Estudios Tecnológicos a Distancia. También, significó la posibilidad de poner en marcha la "profesionalización" de los tecnólogos - para responder a las demandas de los mismos estudiantes - , ya que el decreto definió que los programas de FT también podrían 


\section{investigación}

constituirse en un primer ciclo de la formación universitaria; práctica que se difundió ampliamente en la década de los años ochenta y noventa, a la par de la oferta de especializaciones tecnológicas. Esta última, considerada por el decreto como la alternativa para continuar la formación por vía tecnológica; que presentaba desventajas ya que no eran consideradas como formación de nivel avanzado (posgrado).

Así, en la década de los años ochenta y noventa, el país fue testigo de un crecimiento desordenado de programas de FT y de los ciclos profesionales en todas las áreas del conocimiento y profesiones; pero especialmente en aquellas cuya relación con el desarrollo de tecnologías es cuestionable, a saber: economía, administración, contabilidad, finanzas y demás.

Los años noventa y la promulgación de una nueva ley (30 de 1992), propicia en las universidades públicas la reflexión sobre el concepto de tecnología - desde la perspectiva que propone Quintanilla (1998) como "un conjunto de conocimientos de base científica que permiten describir, explicar, diseñar y aplicar soluciones tecnológicas a problemas prácticos de forma sistemática y racional" (p. 50) - y la incorporación de programas de FT a las tradicionales Facultades de Ingenierías. Además, el establecimiento y consolidación de sedes regionales - para ofrecer a los bachilleres formación superior en sus lugares de origen $\mathrm{y}$, con ello, propiciar el desarrollo regional- $\mathrm{y}$ la descentralización de la oferta educativa a otras localidades de la ciudad capital-perspectiva que asume la UDFJC-, con la institución de una unidad académica que ofrecería ES a sectores desfavorecidos de la capital.

La Facultad Tecnológica asumió su denominación evocando los programas que habría de ofertar; es decir, en línea con el concepto de formación terminal y de corta duración establecido en el país desde finales de los años setenta. Posteriormente, motivada por las propuestas de
Gómez (1995) — pues como dijo un entrevistado en el desarrollo de las entrevistas "fuimos Víctor Manuel Gomezianos" (Docente 39, área ciencias sociales, UDFJC)_, quien asume la tecnología como un campo de conocimientos en el cual se forman tecnólogos e ingenieros y un concepto de FT entendido como la capacidad de investigación, desarrollo e innovación en un área del conocimiento tecnológico, es decir, para la solución de problemas y, por lo tanto, una formación caracterizada por una sólida fundamentación en ciencias básicas - esto controvierte el discurso dominante de los dispositivos de control (DdC) — como bien lo señala Vergara (2007) en uno de los primeros escritos que circuló en la comunidad de docentes, para plantear:

[...] una definición de 'educación tecnológica'. Al respecto se acoge la conceptualización de Víctor Manuel Gómez [la educación tecnológica] ... implica la aplicación de conocimientos científicos para la resolución de problemas concretos. Requiere de la formulación de hipótesis abstractas que pueden ser experimentadas o verificadas, el control o manipulación de sistemática de determinadas variables conceptuales relacionadas con los resultados esperados y la capacidad de diagnóstico e interpretación de los eventos observados. (pp. 3-4)

Ese concepto de FT, la facultad lo extiende a la $\mathrm{FpC} \mathrm{y}$, desde esa última perspectiva, define que la Educación Tecnológica por Ciclos (ETpC) como la interrelación de tres ciclos tecnológicos (Vergara, 2007): el primero que corresponde a los programas que tradicionalmente en el país se denominaban de FT - que ya se venían ofertandoel segundo a las especializaciones tecnológicas que reglamentó la Ley 30; y, el tercero, a una ingeniería con énfasis en un área puntual del conocimiento tecnológico; este último ciclo marcó las discrepancias con los DdC — que representan los intereses de la formación tradicional de ingenieros en el país-, que giraron alrededor de considerar los programas de FT como terminales, de asumir 


\section{investigación}

la ingeniería como un ciclo tecnológico y por lo tanto, el plan de estudios y la denominación con un énfasis - por ejemplo, distribución y redes eléctricas, sistemas avanzados de producción, redes de computadores, mecánica con énfasis en procesos, vías y trasporte, control e instrumentación.

Esta perspectiva de considerar una conceptualización alternativa de FT solo evidencia en el país el esfuerzo de pequeños grupos o, en el mejor de los casos, de algún programa o de una unidad académica, por lograr que la FT adquiera un estatuto conceptual y curricular propio, que le permita profundizar en la construcción del conocimiento tecnológico, desde la perspectiva del desarrollo endógeno y, a la par, contribuir con la ampliación de cobertura - cupos - de la ES; especialmente para sectores poco favorecidos de la población.

Como resultado de la expedición de la Ley 749 , en el país se configuró una superposición de DdC que permite la oferta simultánea programas de FT terminales (Ley 30) y en calidad de segundo ciclo de formación (Ley 749) o nivel tecnológico - como se les denomina actualmente (Decreto 1295) - y programas profesionales tradicionales (Ley 30); además, programas profesionales en calidad de tercer ciclo (Ley 749) o nivel profesional.

Como de hecho ocurre en la UDFJC, que ofrece en la Facultad del Medio Ambiente, programas de FT terminales (Ley 30) y la profesionalización, según la concepción de los años ochenta. En otra (Facultad de Ingeniería) se ofrecen programas tradicionales de formación en ingeniería; y, en la Facultad Tecnológica programas en áreas de la ingeniería desde la concepción de la FIpCP; entre los cuales hay un vínculo curricular mínimo, representado en unas áreas que recientemente la institución reglamentó como transversales; relacionadas con el componente de ciencias básicas y sociohumanísticas.

Lo anterior solo es una evidencia de cómo la ausencia de unas políticas institucionales permea el desarrollo de programas y unidades académicas desde iniciativas personales o de pequeños grupos; que además configura un entramado de tensiones al interior de la institución. Unas tienen que ver, por ejemplo, con el hecho de que actualmente se ofrecen dos programas de ingeniería eléctrica con la misma denominación. Uno en la Facultad Tecnológica, desde la perspectiva de la FIpCP (Decreto 1295) y otra en la Facultad de Ingeniería, desde la perspectiva tradicional (Ley 30); programas que se gestaron por iniciativas separadas y que no comparten presupuestos curriculares, docentes, investigación, entre otros.

El caso de la Facultad Tecnológica de la UDFJC - en el escenario de las universidades públicas observadas - también se configura en un caso sui generis, ya que la mayoría de las universidades públicas no han asumido como propias las disposiciones de la Ley 749, Ley 1188 y el Decreto 1295; la razón fundamental - por ejemplo, en el caso de la Universidad Tecnológica de Pereira-, es que la Facultad de Tecnologías no comparten el concepto de FT referido a un nivel de formación, es decir, que los programas de FT deban constituirse en un escalón hacia la formación en el nivel profesional - ingenierías - ; entonces, continúan ofertando programas de FT terminales (Ley 30) y programas tradicionales en calidad de profesionalización para tecnólogos; el resto de las universidades ofertan programas de FT terminales y dejan a decisión personal de los egresados la continuidad de su proceso de formación profesional en la misma institución —en los programas tradicionales y bajo las condiciones de admisión que estos impongan-, o en cualquier otra institución del país que ofrezca el nivel profesional.

\section{El nivel tecnológico}

Cuando apenas el país y las instituciones empezaban a acomodarse a la Ley 749 y Decreto 2566, se dan a conocer nuevos DdC. La Ley 1188 trajo consigo dos innovaciones: la primera, determinó 


\section{investigación}

que todas las instituciones de ES - incluyendo la universidad - podrían ofrecer FpCP, hasta el nivel profesional y, la segunda, que esa formación podría darse en todos los campos y áreas del conocimiento; se prevé que la enmienda en relación con la participación de la universidad en la oferta de FpCP se da en razón a la tradición que a esa institución le asiste en la oferta de programas de FT y programas de profesionalización —en el marco de lo contemplado en el Decreto-Ley 080 de 1980, como ya se mencionó-, y por supuesto al interés del Gobierno y del MEN de mostrar una mejora en los índices de cobertura de la ES; para lo cual debían contar con la universidad.

En relación con la segunda, parece claro que la restricción que estableció la Ley 749 sobre las áreas en las cuales se podían ofertar FpCP iría en contravía con aquellas que definió el proyecto de fortalecimiento de la educación técnica y tecnológica como prioritarias. Había entonces que garantizar que los DdC no se configurarían en obstáculos para la consecución de dichos fines; por lo tanto, había que derogar lo inicialmente establecido por la Ley 749 .

Por su parte, el decreto precisó que los "programas estructurados por ciclos propedéuticos, son aquellos que se organizan en niveles formativos secuenciales y complementarios" (Ministerio de Educación Nacional, 2010a) y, establece que cada nivel — programa - que integra la propuesta de FpCP, debe conducir a un título que habilite para el desempeño laboral como técnico profesional, tecnólogo o profesional universitario y debe tener una orientación y propuesta metodológica propia que brinde una formación integral en el respectivo nivel, más el componente propedéutico para continuar en el siguiente nivel de formación. Es entonces la expedición de este decreto lo que propicia que la FT deje su identidad en términos de un ciclo de formación y asuma la de nivel tecnológico.
A partir de entonces, los denominados ciclos de formación son dos: el primero, integrado por el nivel técnico profesional y el tecnológico; y el segundo, por el nivel tecnológico y el profesional. Los dos niveles que integran cada ciclo son secuenciales y complementarios entre sí. El controvertido componente propedéutico debe estructurarse en el primer nivel en función de los créditos del primer nivel que hacen parte del segundo y, en el segundo nivel, este puede definirse en términos de disciplinas; especialmente las correspondientes a ciencias básicas.

La ley y el decreto también permitieron corregir - se presume que por la presión que ejercieron las asociaciones de ingenieros y las facultades de ingenierías - otro error o imprecisión de la Ley 749 , cuando señala que los tecnólogos tendrán capacidades de diseño y construcción, y responsabilidades de concepción, dirección y gestión. En ese sentido, la Asociación Colombiana de Ingenieros (ACOFI) señaló que dicha afirmación estaba "generado conflictos debido a algunas pretensiones de los tecnólogos y aún de los técnicos, a través de sus asociaciones gremiales, para realizar funciones propias de los ingenieros" (2010, p. 9). En ese sentido, el Decreto 1295 (artículo 15) fue enfático al señalar que los "niveles técnico profesional y tecnológico deben responder a campos auxiliares, asistenciales, complementarios, innovadores y propositivos de las profesiones, de tal manera que su denominación sea diferenciable y permita una clara distinción de las ocupaciones, disciplinas y profesiones".

Entonces, el tecnólogo no debe ser formado con capacidades relacionadas con el diseño y desarrolo de tecnologías; ya que el objetivo primordial del nivel tecnológico, cuando está incorporado al segundo ciclo, es de servir de formación propedéutica para la ingeniería. Afirmación que se sustenta en lo que el mismo MEN sostiene con respecto a que en un ciclo de formación, el diseño curricular del nivel propedéutico deberá orientarse: 
[...] por el programa de mayor complejidad y duración. Un programa tecnológico por ciclos propedéuticos, deberá entonces orientar los programas técnicos profesionales que albergue y un programa profesional universitario por ciclos propedéuticos deberá orientar los programas tecnológicos y técnicos profesionales que albergue. (Ministerio de Educación Nacional, 2007, p. 16)

En ausencia de capacidades relacionadas con el diseño y desarrollo de tecnologías, como el que prevalece desde los DdC, el tecnólogo no es un tecnólogo y la FT es, simplemente, un nivel de requisito para ingresar a la formación profesional universitaria. Así es como en Colombia, en el actual contexto normativo la FT desaparece. La única alternativa de mantener cierta identidad tecnológica de la formación sería mantener la identidad de los programas de FT como terminales - de conformidad con lo establecido en la Ley 30- lo que significa para el país limitar la PDA de conocimiento tecnológico, con las repercusiones que de ello se derivan tanto para el desarrollo de la investigación en el ámbito académico, como de los beneficios que de ella se desprenden para los sectores industriales y empresariales.

A lo anterior hay que agregar que en el contexto normativo se conservan una sería de dudas adicionales. Algunas de ellas son: ¿por qué seguir considerando que los propósitos de la formación técnica y tecnológica deben seguir siendo los mismos?, ¿el país requiere realmente esos dos niveles de formación con propósito similares, para formar en ocupaciones?, o ¿cuántos programas de nivel tecnológico pueden albergar las ingenierías tradicionales que ofrece la ES en el país? y ¿con base en qué criterios se establecerán las afinidades entre programas de nivel tecnológico y profesional?, más aún si se tienen en cuenta la diversidad de áreas del conocimiento tecnológico muchas de las cuales en sí mismas articulan diferentes áreas del conocimiento tecnológico — por ejemplo la mecatrónica.

\section{investigación}

Por otra parte, ¿cuál es el objetivo de reglamentar los estándares mínimos de calidad para cada nivel - especialmente para el técnico profesional y tecnológico-? si estos deben guiarse simplemente por la ingenierías que los acoge. Es decir, lo que prima no son propiamente los fines de la formación de cada nivel —en este caso del nivel tecnológico- , sino que cada nivel, de menor complejidad — nivel tecnológico- debe garantizar la consecución de los fines del nivel de mayor complejidad — nivel profesional o ingeniería-, como bien señaló uno de los docentes al referirse sobre la orientación del diseño curricular que predomina actualmente:

[...] ligada a esa condición de por ciclos, es que en Colombia nosotros abordamos la formación tecnológica, desde arriba...qué significa desde arriba, desde la universidad pública, desde la institución de más alta jerarquía dentro de las instituciones de educación superior ¡cierto! Y desde la perspectiva de ingeniería, es decir, la experiencia es de ingenieros, cierto y desde ahí se concibe la educación tecnológica hacia abajo. (Docente 35, área electrónica, UDFJC)

Es en razón a las contradicciones internas de los DdC vigentes, que algunas universidades públicas - tal es el caso de la UTP_, no asumen sus conceptos como válidos y optan por continuar en la línea que asumieron en los años ochenta; ofertar programas terminales de FT. Así se refirió un entrevistado al concepto de FT que asume la ley que regula la FpCp:

[...] cuando aparece la Ley 749 nos acercamos a ella, hubo aquí unos tres o cuatro foros alrededor de la situación, y entonces se dijo ¡no!, para nosotros el concepto que está manejado la ley de tecnología no es. Para nosotros no es esa la situación, nosotros seguimos en la línea de fortalecer la parte de la ciencia básica y, la tecnología, para nosotros

Tecnología, ingeniería y ciclos de formación

MIRNA JIRÓN POPOVA 


\section{investigación}

va inclusive un poco más allá de lo que es la ingeniería. (Docente 24, área química, UTP)

A lo anterior hay que añadirle que, en el área de las ingenierías, la tradición del país se inclina a la oferta de programas que propenden a una formación más teórica que práctica; orientada "hacia la formación generalista con alta fundamentación en la estructura básica" (Asociación Colombiana de Facultades de Ingeniería, 2000, p. 22). Por lo tanto, la estructura de los planes de estudio $-\mathrm{y}$ las denominaciones de los programas - no evidencia un área del conocimiento tecnológico de énfasis y, desde esa óptica podrían no considerarse programas de FT. A esa posición de ACOFI entonces responde la Ley 749 - y el actual Decreto 1295-, que reglamenta precisamente una FpCP en programas tradicionales de ingenierías, es decir, una FIpCP. Por ello es que el MEN establece que los estándares de calidad del ciclo profesional corresponden a los de un programa tradicional.

El ciclo de formación profesional (Tercer ciclo propedéutico) de los programas de pregrado en el área de ingeniería, se organizará de acuerdo con el nivel de complejidad correspondiente a esta modalidad educativa y se organizará en concordancia con las características específicas de calidad definidas por el Ministerio de Educación Nacional para los programas de pregrado de Ingeniería. (Ministerio de Educación Nacional, 2003b)

Lo anterior agrega una incoherencia más entre los $\mathrm{DdC}$ vigentes y los presupuesto de la $\mathrm{FpC}$, al establecer que el nivel tecnológico —en calidad de segundo ciclo de formación-, debe ser teóricamente compatibles con el objeto de conocimiento de la ocupación, disciplina o profesión que se pretende desarrollar, es decir, debe evidenciar un énfasis tanto en la estructura del plan de estudios como en la denominación. Entonces, la norma pretende que el primer nivel forme un tecnólogo es un área específica del conocimiento tecnológico y, el segundo nivel un ingeniero tradicional. ${ }^{1}$

Presupuestos cuestionables - desde la concepción de la $\mathrm{FpC}$ - , dado que efectivamente las bondades de este tipo de estrategias curriculares radican precisamente en el hecho de que, al finalizar cada ciclo o nivel de formación, el egresado interactúa con el medio productivo y, por lo tanto, adquiere unos conocimiento que complementan su proceso de formación académica y que se trasforman en experiencia profesional. Al reingresar al siguiente ciclo o nivel, los intereses y la base desde la cual se parte para la formación no puede ser la misma que la de un estudiante que asume una formación tradicional —ingeniería - y que carece de experiencia profesional; figura que asume la ley, ya que desconoce la posibilidad de profundizar en un área del conocimientos tecnológico en segundo ciclo de formación. Es decir, en un programa que sea reconocido como de nivel profesional, que no es el caso de las especializaciones tecnológicas que contempla la Ley 30.

En ese sentido, los DdC rompen la continuidad entre la formación académica y la práctica profesional, cuando ambas están evidentemente interconectadas y, en el caso específico de los programas relacionados con áreas de la tecnología, es evidente la necesidad propiciar ese diálogo. Esa especificidad de la $\mathrm{FpC}$, que debe considerar la experiencia profesional acumulada, es la que lleva a que el segundo ciclo o nivel de formación -

1 En esa línea de argumentación la Asociación Colombiana de Facultades de Ingeniería (2000) sugirió, una nomenclatura de títulos $-\mathrm{y}$ por ende de denominaciones-, para los programas de FT asociados a las ingenierías tradicionales. Por ejemplo, para el caso del área de mecánica (p. 31), en el marco del proyecto, se recomendaron los siguientes programas como asociados a la Ingeniería Mecánica: en mantenimiento aeronáutico, en mantenimiento de equipo biomédico, en mantenimiento industrial, en máquinas y herramientas, en mecánica, en mecánica automotriz, en mecánica industrial, en metalmecánica, en refrigeración y aire acondicionado, en seguridad aeronáutica, en supervisión y mantenimiento de maquinaria industrial, mecánica naviera, en mantenimiento, en máquinas y herramientas, entre otras. 


\section{investigación}

ingeniería - no deba considerarse como la simple diferencia entre los créditos de una ingeniería tradicional y los créditos de un programa de FT, más el componente propedéutico; que en el contexto de los DdC debe responder a las necesidades de la ingeniería tradicional y no a la profundización del conocimiento tecnológico.

Esa contradicción de los DdC es compartida por la Asociación Colombiana de Ingenieros (ACIEM) - por supuesto, con el propósito de defender la formación tradicional de los ingenieros y no de propiciar programas de ingenierías con énfasis-, que afirma que no se puede pretender formar un tecnólogo con énfasis y posteriormente un ingeniero tradicional; para argumentar lo correspondiente, recoge un ejemplo del MEN que ilustra la concepción de FIpCP que promulga la Ley 749.

Por ejemplo, un programa de Ingeniería Mecánica podría organizarse por ciclos de tal manera que después de que un estudiante curse el primer ciclo, éste pueda acceder al título de Técnico Profesional en Mecánica Diesel y a su vez, esté preparado para continuar su formación en un segundo ciclo, después del cual podría acceder al título de Tecnólogo en Mecánica Automotriz y, si así lo desea, continuar su formación en un tercer ciclo para acceder al título de Ingeniero Mecánico. (Ministerio de Educación Nacional, 2007, p. 15)

ACIEM (2010, pp. 10-13) señala entonces que el estudiante, al terminar el segundo ciclo, recibe el título de tecnólogo en mecánica automotriz, pero que existe una enorme diferencia entre la formación requerida por el tecnólogo en mecánica automotriz y la formación que debe tener el ingeniero mecánico; y esto lo sustenta en las diferencias entre los propósitos de formación que persigue cada uno de los componentes o áreas de formación, así sus denominaciones sean similares. A ello agrega que, mientras la profesión de la ingeniería tiene sólidas bases científicas, no sucede lo mismo con el ciclo tecnológico - por supuesto, si se trata de los programas que asumen los propósitos de formación desde la perspectiva de desarrollar destrezas y habilidades, es decir, desde el concepto que se consolidó con la emergencia de la modalidad-, por lo tanto, considera que esos programas no hacen parte de la ingeniería; así aborde temas propios de la profesión, con enfoques diferentes. Entonces, la tecnología en mecánica automotriz no puede considerarse como un camino recorrido dentro de la formación en ingeniería mecánica.

De esa manera, los DdC no extienden el concepto de FT a las ingenierías - y de hecho no pueden, dado que la FT se reduce a un nivel de formación previo al profesional, de tres años de duración, que conduce al título de tecnólogo en cualquier disciplina o profesión-, de lo contrario las ingenierías pondrían en evidencia el área de profundización en el conocimiento tecnológico - en la denominación y en la estructura del plan de estudios, como por ejemplo: robótica, microelectrónica, redes eléctricas, energías renovables, bioingeniería, procesos de manufactura, vías y trasporte, sistemas de producción, mantenimiento industrial, plásticos, textil, instrumentación, entre otras - En cuyo caso sería correcto denominar la propuesta de $\mathrm{FpC}$ como formación tecnológica por ciclos (FTpC) o ETpC; perspectiva que asumió la Facultad Tecnológica de la UDFJC.

En relación con las diferencias entre asumir la FT desde la perspectiva de los DdC —o sea desde la ingeniería tradicional-, o desde aquella que propende a la profundización del conocimiento tecnológico, un directivo entrevistado en la UTP mencionó:

¿Entones un ingeniero tradicional no hace tecnología?, claro el ingeniero está trabajando en tecnología, sí, pero es que el ingeniero nuestro se quedó en la mirada muy limitada a ciertas áreas... entonces, el programa de tecnología mecánica está pensan- 


\section{investigación}

do en un programa de ingeniería en manufactura y, usted puede preguntar ¿qué tiene el programas de manufactura frente al programa que tenemos en la Facultad de Ingeniería Mecánica?, que ellos no se centran en manufactura. En esa línea tienen que diseñar el producto, entregarlo y hacer que cumpla una función en el mercado. Así, cuando los tecnólogos mecánicos lleguen a la producción tienen que ser fuertes en manufactura en el país y en el hacer; un ingeniero con un conocimiento tecnológico muy fuerte, en las últimas tecnologías, en las tecnologías de punta. (Docente 24, área química, UTP)

\section{De la educación tecnológica por ciclos a ingenierías por ciclos propedéuticos: la armonización con los dispositivos de control}

La emergencia del sistema de aseguramiento de la calidad de la ES significó para la Facultad Tecnológica el inicio de un proceso de transición de la ETpC a la FIpCP. Un primer paso en ese camino se configuró con la emergencia del primer decreto que introdujo estándares de calidad para programas organizados por ciclos propedéuticos (Decreto 2566).

Sin asumir la tarea de analizar a fondo lo contenido en el decreto, especialmente en lo que a la concepción de lo propedéutico se refiere - por supuesto en razón a la carencia de conceptualización sólida en ese sentido - , la Facultad consideró que este reflejaba el espíritu de la propuesta de formación y, ajena a su suerte, decide iniciar el proceso de solicitud de estándares de calidad; decisión que se considera marca el nacimiento de la FIpC; por lo tanto, debía asegurar que "en ambos ciclos se está desarrollando las competencias propias de un ingeniero mecánico" (Proyecto Curricular de Tecnología e Ingeniería Mecánica, 2005, p. 8) y para ello había que asumir como propio lo dispuesto en la Resolución 3466 sobre la estructura de los planes de estudioso de los programas de ingenierías, organizados en ciclos; el proceso dejó como saldo el cambio total de las denominaciones de los programas — que asumieron denominaciones tradicionales -2 y una estructura de los planes de estudio que, indiscutiblemente, ya no respondía a laETpC, más bien a programas de FIpC.

Pese a los traspiés, la Facultad siguió adelante con miras a materializar una propuesta que ya no estaba a su alcance, pero a la cual seguía aferrada: una ETpC. Para esa época (2006- 2009) la institución pasaba por una nueva coyuntura de reforma interna, que afectaría tanto a los programas de ingenierías como a los de FT, como resultado de la necesidad, ahora institucional, de responder a las demandas de DdC; pues ya habían sido reglamentados los estándares para la totalidad de las áreas del conocimiento y profesionales y ello implicaba que la institución iniciaría la presentación masiva de los programas de la totalidad de las facultades.

La universidad asume entonces que debe acometer una reorganización curricular institucional, para lo cual aprueba (2006) formalmente el paso de los planes de estudio a créditos académicos y la definición de perfiles en términos de competencias. En el marco de la reglamentación expedida por el Consejo Académico, los programas de FT fueron denominados de Nivel Profesional Tecnológico y su duración en créditos de 96 a 108.

Además de asumir los créditos y las competencias como referentes para estructurar los planes de estudio de los programas de pregrado, la institución establece un componente trasversal a los programas de pregrado de la universidad - que si bien desde esa época se vienen poniendo en mar-

2 Ingeniería civil, Ingeniería eléctrica, Ingeniería mecánica, Ingeniería en telecomunicaciones, Ingeniería de producción-denominación se sugirió en reunión conjunta entre el MEN y los directivos de la Facultad Tecnológica teniendo en cuenta que la Facultad de Ingeniería contaba con un programa de Ingeniería Industrial- e Ingeniería en control. 


\section{investigación}

cha, sólo se formalizó en el 2011—, que obligó a reformar los programas y, acorde con los propósitos que ostentaba la FT en el marco de la FIpC - servir de fundamentación a las ingenierías-, hubo que sacrificar espacios académicos del componente de aplicadas de la profesión, o en su defecto, reducir su peso en créditos, para acomodar las cátedras, segunda lengua, los nuevos espacios académicos de ciencias básicas y humanidades.

En correspondencia con ese nuevo escenario curricular, los perfiles muestran una reducción significativa de los ámbitos de acción tecnológica de los tecnólogos; lo que es completamente coherente con el hecho de que el propósito prioritario de estos programas es fundamentar la formación en ingeniería. Tal y como afirma un entrevistado, esta situación solo evidencia que la Facultad ha venido desistiendo de su propuesta de formación original, para cumplir con lo establecido en las normas institucionales que también ven en la FT un ciclo de fundamentación de la ingeniería y, por lo tanto ejercen control sobre la estructura de los planes de estudio, a manera de:

[...] una camisa de fuerza curricular, donde nos han traído unas cátedras, donde tenemos una cantidad de horas lectivas, el tema de los créditos. Entonces el área disciplinar no se mueve libremente, ha estado limitada y, en ese límite, hemos tenido que hacer muchas cosas, abordar varias estrategias para cubrir todo el cuerpo de conocimientos que requiere un tecnólogo para graduarse. A mí consideración, valdría la pena hacer un rediseño de esa camisa de fuerza curricular que nos ha impuesto la universidad. (Docente 42, área electrónica, UDFJC)

\section{La reforma exprés}

Si bien las bondades de la propuesta académica de la facultad en relación con el impacto social en el sector de influencia no pueden desconocerse. Hay que reconocer que el concepto era uno de los puntos débiles, dado que se conjugaban posiciones particulares, como bien lo señaló un docente del área de civil "si hablamos de la propuesta de civil, que difiere de la propuesta industrial y además que la de electrónica, eso no, ni por lo menos hubiera eso, pero es que ni siquiera lo hay, pareciera haber una tendencia de dos" (Docente 6).

En este sentido, tanto la conceptualización como también se observó en otras universidades públicas-, como el trabajo curricular que de ello se deriva, son casi que privativos de unos pocos docentes y no obedecen a unos lineamientos o políticas institucionales. Así entonces, los entrevistados tiene razón cuando afirman que la facultad:

[...] institucionalmente no tiene unos lineamientos o una posición clara para el modelo de ciclos, nosotros institucionalmente no sabemos qué es un tecnólogo, no hay una política institucional y ¿cómo se llama eso en los documentos? Sí, los documentos que digan mire es que la tecnológica, en todos los proyectos tenemos este ciclo uno que se compone de esto, esto y esto. Su perfil es este y este y este, y esperamos que el estudiante sea esto y esto y esto. (Docente 30, área civil, UPTC)

En otras palabras, no hay uno documento institucional que dé cuenta del concepto de tecnología, de FT, de FIpCP, ni sobre el componente propedéutico. Lo que estableció fueron unas prácticas, en palabras de un entrevistado, se instauró "un discurso muy etnográfico, muy de lo que se hace. Y de eso cada uno da cuenta" (Docente 39, áreas ciencias sociales, UDFJC). Así, cuando se preguntó al docente sobre el concepto de FT, respondió:

[...] cada uno tiene un concepto diferente de lo que es educación tecnológica, fíjate como apenas hay algunos pinitos hechos por algunos docentes, principalmente pues los que pertenecemos al Grupo de Investigación en Educación Tecnológica por 


\section{investigación}

Ciclos y, los que en algún momento pertenecieron, pero de ahí para allá nadie teoriza sobre la educación tecnológica. (Docente 39, área ciencias sociales, UDFJC)

Las pocas puntadas que dio la facultad fueron conceptualmente pobres - como bien lo hizo ver uno de los entrevistados, "habían unas ideas básicas, unas propuestas preliminares, pero que cuando sacudimos ese escalón, no encontramos evidencias de modelo de formación por ciclos" (Docente 44, área ciencias sociales, UDFJC)-, por lo tanto, no despejan las dudas de los actores sociales; en cuanto a los propósitos de formación y en especial en lo que respecta a los alcances del nivel tecnológico y su articulación con el nivel de ingeniería; lo cual, aunado a los sucesivos cambios de los DdC y las implicaciones que tuvo que asumir la facultad, configuró una suerte de caja negra.

Así, lo hicieron saber algunos entrevistados. Uno de ellos mencionó no saber "bien cómo es la cuestión del modelo por ciclos, digamos desde su visión como ya más epistemológica, digamos filosófica, la mayoría de profesores, hasta los de cátedra ni saben qué es eso" (Docente 23, área civil, UDFJC), entonces "en general los profesores, no saben que existe educación por ciclos, empezar a meterlos en ese rol es un trabajo maratónico, no se logra de la noche a la mañana ... desafortunadamente no lo hemos logrado hacer con todos." (Docente 42, área electrónica, UDFJC), por el contrario solo con unos pocos y, la facultad no ofrece "inducción para los docentes es nula, más allá de lo que el coordinador puede comentarte en el momento en que entrega la carga, bien sea a un profesor de hora cátedra o a uno de planta, no es formal la inducción" (Docente 22, área civil, UDFJC) y, tampoco hay seguridad en que se formen tecnólogos. A ese respecto un docente del área de civil, cuestionó:

[...] ¿Verdaderamente estemos formando tecnólogos? Que la malla curricular esta diseñada para formar un tecnólogo, eso no lo cree ninguno. De pronto si uno mira la malla curricular, de pronto el muchacho tiene algo tecnológico en lo que nosotros llamamos Materiales, Construcción I, Construcción II, Construcción III, pero volverse específico, ya hablando de un perfil profesional desde lo tecnológico. (Docente 23, área civil, UDFJC)

Entonces, para acometer con éxito el proceso de registro calificado, la facultad aceptó que desarrollaba un segundo ciclo de formación que incorpora dos niveles uno tecnológico y otro profesional; en el marco de un acuerdo que denominó la propuesta de formación con una denominación discreta: "Modelo Educativo" (Consejo Superior Universitario, 2010), en razón a que los ponentes del acuerdo no tenían suficientes argumentos para dotar de identidad la propuesta o no quisieron asumir los costos políticos de tal decisión; en razón a las tensiones que se generaron al interior de la Facultad y que motivaron a la opción de pensar en alternativas como, por ejemplo, los docentes del área civil proponía olvidar por completo la FIpC y la FIpCP.

Otro docente, del área de electrónica, planteó volver a los presupuestos de origen de la Facultad, cuando la FT — es decir, el primer ciclo tecnológico- era "lo primordial y, la ingeniería si nos la quitan hombre finalmente no hay problema porque esto es una Facultad Tecnológica" (Docente 45, área electrónica, UDFJC), pero era la minoría la que pensaba así "siete u ocho profesores en la facultad que nos interesa que se discuta si debemos seguir sacando ingenieros por ciclos o si debemos darle duro a la formación de tecnólogos" (Docente 35, área electrónica, UDFJC), para la mayoría — que se contraponían a la idea anterior-, la solución para la facultad era aunar esfuerzos para fortalecer las ingenierías desde la concepción tradicional, así ello signifique, en palabras de un docente del área civil, ofertar: 


\section{investigación}

[...] la ingeniería por un lado y la tecnología por otro. Es una discusión que al interior del proyecto curricular hemos dado, en consejos curriculares ampliados donde ha habido espacio para que todos hablemos y en conjunto, el $100 \%$, hemos llegado a la conclusión de que sí queremos fortalecer la ingeniería se nos debilita la tecnología y viceversa. (Docente 22, área civil, UDFJC)

En suma, posiciones divergentes que coinciden únicamente sobre la urgente necesidad de "analizar muy bien si lo que queremos es irnos por la vía de la ley o si lo que queremos retomar el proceso que se gestó aquí desde hace muchos años" (Docente 43). Y es ahora cuando los docentes reconocen que la facultad y la universidad no participaron de los espacios de discusión de los DdC, entonces no se podía esperar que "nos tuvieran en cuenta cuando las hicieron y, nosotros llevamos muchos años formando ingenieros aquí" (Docente 41, área civil, UDFJC) y la universidad tampoco se preocupó por:

[...] participar de esas mesas y, en la legislación. Es decir, el problema es que otros están tomando decisiones que deberíamos liderar nosotros; nos estamos haciendo a un lado y otros son los que toman las decisiones y qué terminamos haciendo, acomodándonos a la ley, que es lo que estamos haciendo en este momento, tratando de sumarnos a la ley. Porque nosotros no hemos hecho la tarea y cuando digo todos, nosotros pues es toda la universidad en general. (Docente 42, área electrónica, UDFJC)

El acuerdo entonces no fue más que un esfuerzo de tipo operativo para responder a las demandas externas de las instituciones de control. Acuerdo al que hay que abonar que recoge el único punto de acuerdo, que el nivel tecnológico es propedéutico con respecto a la ingeniería afín. Sobre esta particularidad: propedéutica del nivel tecnológico, es oportuno añadir que, la afirmación es válida desde la perspectiva intrínseca de lo propedéutico que asumía la facultad; y no era una característica trasversal a la totalidad de los programas. Algunos incorporaban los contenidos propedéuticos en asignaturas del nivel de ingeniería $y$, otros, los ubicaban en los dos niveles, como afirmó uno de los entrevistados "dentro de nuestro programa tecnológico o dentro de nuestro programa de ingeniería dependiendo del proyecto curricular, sin necesidad de devolverse ni de volver a ver otro tipo de cosas" (Docente 26, área eléctrica, UDFJC); entonces el acuerdo introduce una imprecisión, que refleja el desconocimiento - de los gestores-, de la realidad curricular.

A lo anterior hay que agregar que, desde la perspectiva extrínseca que define la norma, la afirmación tampoco es válida, ya que debe ser propedéutico el nivel que recibe; la ingeniería es propedéutica al nivel tecnológico. Decir lo contrario es asumir de antemano que todos los tecnólogos continuarán su proceso de formación y que, además, lo harán de forma casi inmediata. El componente propedéutico cobra pertinencia cuando efectivamente hay certeza de la continuidad de los estudios en nivel de formación; considerar la tecnología propedéutica equivale a decir que, para que el estudiante reciba su grado, debe cursar los créditos propedéuticos, lo que nuevamente no es correcto en razón a que ese componente no aporta a la formación del perfil de egreso del tecnólogo.

Entonces, pese a declarar la armonización, el MEN no renueva los registros de los programas de ingeniería solicitados en razón a que "la presentación del componente propedéutico en la estructura curricular y las asignaturas expuestas no poseen carácter propedéutico" (Ministerio de Educación Nacional, 2010a, p. 1) -lo que demuestra cómo en el país los DdC permiten ejercer control sobre la ES-, y efectivamente la afirmación era correcta como señaló un docente toda vez que: 


\section{investigación}

[...] no somos ciclos propedéuticos, así adaptemos los documentos y tratemos de apuntar y de ponerle pinzas a la cosa, en este momento no somos ciclos propedéuticos, hemos defendido nuestro modelo de formación por ciclos que es diferente. Nuestra formación por ciclos qué implica: hacer una homologación, un traslado de tecnología a ingeniería, es un sistema de homologación que para nada tiene que ver con ciclos propedéuticos y un ensamble de un grupo de asignaturas, para complementar una malla curricular de un ingeniero. (Docente 42, área electrónica, UDFJC)

Esta coyuntura llevó a la facultad a tomar una decisión pragmática, adelantar una reforma exprés para poner en evidencia del MEN, como dijo un docente, que "nos piden modificaciones, adaptaciones, ipues hombre hay que hacerlas!" (Docente 45). Así el Consejo Académico (2011), asumió la tarea que había dejado pendiente desde 1998, reglamentar algunos aspectos de la $\mathrm{FpC}$, que definió como una expresión de flexibilidad curricular, en la cual dos ciclos - aquí el término ciclo que utiliza el texto de la resolución hace referencia al término nivel expresado en la norma- de formación se disponen en un orden secuencial predefinido, con el propósito de acrecentar la complejidad y profundización del conocimiento en el tiempo. Es decir, que un ciclo de formación es un conjunto de conocimientos organizados en un plan de estudios — de un programa académico-, que conducen a un título profesional de pregrado.

El primer ciclo de la secuencia es propedéutico al segundo y, este último, complementario al primero. Así, ciclo tecnológico antecede al ciclo de ingeniería en términos de la complejidad y profundización del conocimiento; el ciclo de ingeniería complementa - también en términos de la complejidad y profundización del conocimiento- el ciclo tecnológico y el controvertido componente propedéutico -definido como un conjunto de asignaturas que garantizan la continuidad de la formación, del ciclo tecnológico al ciclo de ingeniería - podrán incluir asignaturas de las ciencias básicas, sociohumanísticas, económico, administrativas, básicas de la profesión o aplicadas de la profesión; posición bastante amplia, que tuvo como telón de fondo la intención de no generar controversias entre los docentes de las diferentes áreas.

Además, el Consejo Académico, se vio obligado a interpretar algunos de los aspectos contenidos en el reglamento estudiantil, para poder administrar los componentes propedéuticos de los programas, como bien manifestó un docente "por sí pregunta el MEN “¿dónde está reglamentado eso? Por ejemplo, en ¿qué Consejo Académico o qué ente a nivel digamos regulatorio dentro de la universidad se aprobó? ¿Dónde se sustenta la creación de ese componente propedéutico?" (Docente 45, área electrónica, UDFJC) y la Facultad tuvo que admitir que:

[...] la lectura e interpretación que se realizó del Decreto 2566 de 2003 (artículo 4 y 24) en nuestra Facultad, aunada a nuestra práctica, no entendió lo propedéutico diferenciado del plan curricular. Es por ello que los documentos puestos a consideración para la solicitud del registro calificado del programa de Ingeniería Mecánica por Ciclos Propedéuticos y, específicamente lo correspondiente a Aspectos Curriculares no contemplaron la presentación de un componente propedéutico diferenciado curricularmente más sí, intrínseco y flexible en la integralidad de nuestros niveles de formación. Así, sobre el particular la Facultad Tecnológica asume que entendió el significado de lo propedéutico de manera distinta pero no contradictoria con la norma. (Rectoría, 2010, p. 2)

En suma, todo un acontecimiento institucional, acompañado de una secuencia de transformaciones indispensables para cumplir con los DdC, llevó a la reforma de los planes de estudio; a costa de permear la propuesta de la facultad por las 


\section{investigación}

políticas ministeriales y gubernamentales, que a lo largo de la historia dispusieron que la Facultad tenía que desvirtuar los presupuestos de la ETpC y asumirse en relación con lo que en la actualidad, después de todo este proceso de reformas es, como señaló un docente del área de sistemas, "una ingeniería por ciclos propedéuticos que no propende por formar tecnólogos, capacitamos a nuestros tecnólogos para ser ingenieros, para ingresar al nivel de ingeniería y, graduarse como ingenieros" (Docente 26, área eléctrica, UDFJC).

\section{CONCLUSIONES}

La pregunta por la incidencia de las normas y, especialmente, la reglamentación de la FpC en el concepto de FT, tiene respuestas diferenciadas según la perspectiva de cada institución. En el caso de los programas que históricamente asumen una visión instrumental de la tecnología, el concepto no contradice con lo que establecen los DdC vigentes. En aquellos programas que toman un concepto - como en la Facultad de Tecnologías de la UTP y la Facultad Tecnológica de la UDFJC - desde los presupuestos modernos de tecnología y estrechamente articulado con la formación en áreas relacionadas con la producción industrial y los fundamentos teóricos de las ciencias para el desarrollo de actividades de diseño e investigación, no asumen $-\mathrm{y}$ las normas por ahora no los obligan - lo dispuesto en los DdC. Pero esos conceptos no se comparten en el ámbito institucional $-\mathrm{y}$ menos en el nacional—, pues el interés de quienes ostentan el poder normativo e institucional es mantener el orden establecido.

Entonces, se evidenció un impacto significativo de la reglamentación de la FpCP fue en la Facultad Tecnológica de la UDFJC que, desde inicios de los años 2000, inició el tránsito de la propuesta de $\mathrm{ETpC}$ - lo cual no pudo concretar en razón a que los DdC se interpusieron en la consecución de esos fines-, en razón, primero, a las divergencias con los estándares de calidad para programas tradicionales de ingeniería (Decreto 792), segundo, con la perspectiva de la FIpCP de los DdC (Ley 749) y, tercero, las dificultades asociadas a la interpretación del componente propedéutico (Decreto 2566 y 1295).

Discrepancias que causaron la armonización definitiva de la propuesta de ETpC con lo instituido en los DdC y, con ello, la aceptación de la FIpCP y lo propedéutico como un componente curricular extrínseco al plan de estudios. Decisión que se justifica en la premura de garantizar a la continuidad académica de la propuesta de formación; así el costo de esa decisión fuese desfigurar los presupuestos originales de ETpC. Cabe anotar, que algunos miembros de la comunidad sugirieron asumir la perspectiva de profesionalización de los años ochenta, sin embargo, esta posibilidad recibió un sinnúmero de cuestionamientos - por parte de un sector de docente que continua convencido en la pertinencia de la propuesta de la FpC así sea en perspectiva con los DdC- y no fue considerada.

En el escenario de armonización, la construcción de un concepto, el establecimiento de lineamientos para la estructuración de los planes de estudio - específicamente en lo que respecta al componente propedéutico - y la participación de la institución en aquellos escenarios nacionales en los que se debaten y definen las políticas, queda relegado a un segundo o tercer plano; más aún cuando el propósito del acuerdo de armonización se cumplió, ya que la institución recibió seis de las siete resoluciones que acreditan la oferta de los programas de ingeniería por ciclos propedéuticos. Es de suponer que en el presente las tensiones han cedido y van quedando en el olvido y, con ellas, las reflexiones, cuestionamiento y propuestas que suscitaron entre los actores sociales.

Cabe señalar que, el actual contexto normativo no es propició para fortalecer un concepto alternativo de FT, uno que la dote de identidad propia, ya que el objetivo primario - en el marco de la FIpCP- 


\section{investigación}

es garantizar la fundamentación que requiere en nivel de ingeniería. A lo anterior hay que agregar que los programas de nivel tecnológico pueden ofrecerse en cualquier campo del conocimiento y profesión, en varios tipos de instituciones. Es decir, que lo tecnológico está referido al nivel de formación, en líneas con la tradición normativa del país; coyuntura que debe poner en cuestión las razones por las cuáles — por vía de los DdCse instituyen unos conceptos con menoscabo de otros, seguramente no menos legítimos.

En consecuencia, en el país se hace inaplazable propiciar y fortalecer el debate conceptual - $\tan$ to dentro del MEN como de las instituciones de ES-, desde varios escenarios posibles. Uno de ellos, consolidar la FT como una modalidad válida de formación profesional, con salidas propias por vía tecnológica-, a los niveles de formación avanzada — posgrados_-; sin necesidad de pasar por denominado nivel profesional —ingenieríacomo ocurre actualmente. Lo que implicaría incrementar por lo menos en un año la duración actual de los estudios y definir unos propósitos de formación articulados al diseño, adecuación, adaptación y, en especial, a la producción de unas tecnologías que respondan a las necesidades del país y de la región.

Otro escenario, sería contar únicamente con dos niveles de formación en el pregrado, ya que la experiencia del país demuestra que la coexistencia de los niveles técnico profesional y tecnológico no fue un decisión acertada; como tampoco lo fue la oferta de especializaciones tecnológicas, las cuales parece que tienen a desaparecer ya que hoy la mayoría de los tecnólogos optan por el ingreso a los programas profesionales — pues son los que prefieren ofrecer las instituciones de educación superior, pues la misma Ley 30 desestimuló estos programas al darle un estatus de formación de pregrado, y no de especializaciones; ${ }^{3}$

3 A ello se asume que se debe la disminución del número de programas de especialización. Una consulta reciente

86

Tecnura | Vol. 18 | No. 40 | abril - junio, 2014 título que no otorga tarjeta profesional - trámite para el cual es indispensable acreditar el de profesional (ingeniero)-, limita la acción profesional de los especialistas tecnológicos y configura una contrariedad entre los niveles de formación que establecen los DdC y las normas que regulan el ejercicio de la profesión en ingeniería.

Entonces, el segundo escenario, incluiría un primer nivel que podría denominarse técnico superior que además se utiliza en buena parte de los países de la región-, y otro profesional. Es decir, volver al esquema de dos niveles de formación en el pregrado - tal y como lo propusieron las misiones nacionales que se llevaron a cabo en la primera parte de la década de los años noventa y también el Dr. Víctor Manuel Gómez-. A la par habría que establecer claramente los propósitos de la formación técnica superior - que bien podría ser la que actualmente establecen los DdC (Decreto 1295) - y acercar la formación actual de los ingenieros tradicionales a la solución de problemas tecnológicos, es decir, a un concepto moderno de FT; este escenario, seguramente sería aceptado con menos resistencia por las agremiaciones de ingenieros y por las facultades de ingeniería del país.

Cabe anotar, que cualquiera de los dos escenarios propuestos debe acompañarse de un cambio en la denominación del título — que puede ser de ingeniero con énfasis en el primer caso o técnico superior en el segundo-, dado que el actual título "tecnólogo" se vincula tanto a la subvaloración académica como social y no es aplicable a la totalidad de áreas del conocimiento.

\section{FINANCIAMIENTO}

El proyecto de investigación La Formación Tecnológica en Colombia (1990-2008), se articula al

del SNIES - en área de la Ingeniería, arquitectura, urbanismo y afines - , registra 89 programa de esos programas, de los cuales 19 están activos y 70 inactivos 


\section{investigación}

macro proyecto Educación Superior y Representaciones Sociales (Doctorado Interinstitucional en Educación (DIE-UD), Línea de Investigación Historia, Pedagogía y Educación Comparada). El proyecto fue avalado - institucionalizado - ante el Centro de Investigaciones y Desarrollo Científico de la Universidad Distrital Francisco José de Caldas en calidad de tesis de doctorado, en marzo de 2010. La financiación del proyecto se llevó a cabo con recursos propios.

\section{REFERENCIAS}

Atcon, R. (2009). La universidad latinoamericana: claves para un enfoque conjunto de desarrollo coordinado social, económico y educativo en América Latina (edición digital por Christin Hernández Amaya ed.). (Banco Mundial, Ed.) Bogotá: Universidad Nacional de Colombia. Acceso: 2011.

Asociación Colombiana de Facultades de Ingeniería. (2000). Nomenclatura de titulos en la formación técnica, profesional, tecnológica y de ingeniería en Colombia. Bogotá: Instituto Colombiano para el Fomento de la Educación Superior.

Asociación Colombiana de Ingenieros. (2010). Reflexiones sobre la formación por ciclos propedéuticos. Recuperado de http://registrocalificado.files.wordpress.com/2012/02/ aciem, ciclos, propedeuticos $1 . p d f$

Congreso de la República. (19 de julio de 2002). Diario Oficial 44.872. Ley 749 "por la cual se organiza el servicio público en las modalidades de formación técnica profesional y tecnológica y se dictan otras disposiciones. Bogotá: Congreso de la República.

Congreso de la República. (28 de diciembre de 1992). Diario Oficial 40.700. Ley 30 "por el cual se organiza el servicio público de la educación superior". Bogotá: Congreso de la República.
Consejo Académico. (2011). Resolución No.048 "por la cual se reglamentan aspectos de la formación por ciclos en la Facultad Tecnológica de la Universidad Distrital Francisco José de Caldas. 3. Bogotá: Universidad Distrital Francisco José de Caldas.

Consejo Superior Universitario. (2010). Acuerdo No.003 "por el cual se precisa y armoniza la oferta educativa en la Facultad Tecnológica”. Bogotá: Universidad Distrital Francisco José de Caldas.

García, B. (2008). Sobre el método: semántica cualitativa, historia de las ideas y análisis de contenido. Bogotá: (s.d.).

Gómez, V. (1995). La educación tecnológica en Colombia ¿Educación Terminal o primer ciclo de las ingenierías y las ciencias? Bogotá: Ediciones de la Universidad Nacional de Colombia.

Ministerio de Educación Nacional. (2010a). Resolución No.8504 "por medio de la cual se resuelve la solicitud de renovación de registro calificado del programa de Ingeniería Mecánica de la Universidad Distrital Francisco José de Caldas para ser ofrecido en la modalidad de ciclos propedéuticos". Bogotá: Ministerio de Educación Nacional.

Ministerio de Educación Nacional. (2010b). Decreto No.1295 "por el cual se reglamenta el 


\section{investigación}

registro calificado de que trata la Ley 1188 de 2008 y la oferta y desarrollo de programas académicos de educación superior. Bogotá: Ministerio de Educación Nacional.

Ministerio de Educación Nacional. (2007b). Formación por ciclos propedéuticos. Bogotá: Ministerio de Educación Nacional. Acceso: 2009.

Vergara, R. (2007). Educación por ciclos: una visión de futuro y una necesidad en el contexto educativo colombiano. Tecnura, 4(6), 3-10. Recuperado de http://tecnura.udistrital.edu. co/ojs/index.php/revista/article/view/81/83

Presidencia de la República. (9 de octubre de 2003). Decreto No.2566 "por el cual se es- tablecen las condiciones mínimas de calidad y demás requisitos para el ofrecimiento $y$ desarrollo de programas académicos de educación superior y se dictan otras disposiciones". Bogotá: Ministerio de Educación Nacional.

Presidencia de la República. (22 de enero de 1980). Diario Oficial 35.465. Decreto-Ley 080 "por la cual se organiza el sistema de educación post-secundaria”. Bogotá: Presidencia de la República.

Quintanilla, M. (2005). Tecnología: un enfoque filosófico y otros ensayos de la filosofía de la tecnología ( $1^{\mathrm{a}}$ ed.). México: Fondo de Cultura Económico. 